\title{
Parcours de la francophonie en Iran : une francophonie latente
}

\author{
Mahmoud Reza Gashmardi \\ Université d'Isfahan \\ Ebrahim Salimikouchi \\ Université d'Isfahan
}

\section{Introduction}

Après plusieurs siècles de présence du français en Iran, nous pouvons dire que la francophonie dans son sens courant, c'est-à-dire d'usage total ou partiel du français, n'existe pas dans la société iranienne. Cela peut s'expliquer par le fait que le français n'est pas une langue véhiculaire en Iran comme c'est le cas en Afrique du Nord. En tout cas, le français persiste dans certaines couches de la haute société iranienne et dans certaines universités et établissements scolaires et culturels des grandes villes.

Malgré tout, on ne pourrait pas dénier catégoriquement une présence particulière de la langue française, sous une autre forme, au sein de la société iranienne. Pour préciser et définir une telle situation, on pourrait avoir recours à la notion de "francophonie latente » où une «langue est significative pour des raisons historiques ou culturelles» (Grand Dictionnaire Terminologique). Elle se définit par une présence en deux sphères, d'abord linguistique par l'intermédiaire de la traduction sous forme de l'emprunt et du calque, et ensuite culturelle sous l'influence des courants littéraires et intellectuels. Cette francophonie culturelle latente s'adresse majoritairement aux intellectuels iraniens par le biais d'un grand nombre d'ouvrages en sciences humaines traduits en persan. Les intellectuels et les universitaires iraniens se familiarisent donc avec la culture francophone sans parfois même connaitre le français.

La didactique du Français Langue Étrangère (FLE) en Iran joue pour la diffusion du français un rôle important dans la francophonie dans son sens courant, et, pour les traducteurs formés dans les universités iraniennes, dans la francophonie latente. La didactique du FLE en Iran peut être examinée selon divers points de vue. Un acte éducatif, selon les formulations différentes, chacune basée sur ses propres principes théoriques, peut se composer selon trois axes principaux : «méthode, professeur, élève » (Girard 11), ou «savoir, professeur, élève» (Houssaye 13-24), ou «objet (langue et culture), agent (personnes, processus, moyens), sujet (apprenant de L2)»(Germain 10-14). Or, il ne s'est pas avéré inutile d'étudier les trois facteurs liés à la didactique du français en Iran pour présenter l'état actuel de son enseignement, tout en évitant, bien entendu, d'ouvrir un débat entièrement épistémologique et théorique.

Pour faire une mise au point sur la francophonie en Iran, nous étudierons son passé et son présent. Nous traiterons d'abord de l'histoire de la francophonie, à savoir le début de 
l'apparition et de l'enseignement du français ainsi que de sa propagation dans la société iranienne. Puis, pour présenter l'état actuel de l'enseignement du FLE en Iran, nous aborderons les trois composantes de l'acte didactique (l'agent, le sujet et l'objet). Pour évaluer la présence de la francophonie latente, nous étudierons, en dernier lieu, ses traces linguistiques, sa présence culturelle et sa réception littéraire en Iran.

\section{Genèse de la francophonie en Iran}

Le XVII siècle a marqué une époque où l'Église a entrepris une tâche importante en vue d'envoyer ses missionnaires en Perse. Ces missionnaires français étaient interprètes, conseillers et ambassadeurs. Parmi eux, Jacques Dutertre, en religion il s'appelle le Père Capucin Raphaël du Mans, qui meurt en Perse après y avoir vécu cinquante-deux ans (1644-1696), était un véritable ambassadeur de France à la Cour d'Ispahan. C'était un génie des langues. Il avait une connaissance exceptionnelle du persan et du turc (Tavernier 211216). Il a pris, pour la première fois, l'initiative d'enseigner le français à la Cour royale d'Ispahan sous le Chah Abbas II. Cela peut être considéré comme le début d'une tâche éducative informelle. La résultante de cette entreprise culturelle fut que, quelques années plus tard, Dieulafoy a pu admirer la parfaite connaissance de la langue française du «Roi » persan lors de son audience avec le Chah Naseroddin, ainsi que celle de la majorité des princes de la dynastie Qadjar ${ }^{1}$ (103 \& 125).

Durant le règne de Naseroddin Chah (1848-1896), on a commencé à traduire des livres scientifiques, littéraires et historiques. À l'initiative et avec la bienveillance du respectueux et grand homme d'état, le chancelier Amir-Kabir, on a fait construire en 1851 Dar ol-Fonun, école polytechnique, où l'enseignement scientifique se faisait en français et la majorité des professeurs étaient français. Sept ans plus tard, étant donné que le plus grand nombre des étudiants avait appris le français, le gouvernement a envoyé les quarantedeux meilleurs élèves de cet établissement en France pour qu'ils y continuent leurs études. La plupart d'entre eux ont étudié la médecine. Ces anciens étudiants allaient peu à peu remplacer les professeurs étrangers. De cette façon, par le biais de cette école, le français est devenu, pour de nombreuses années, la langue de l'enseignement supérieur.

Comme les Européens résidant en Perse avaient obtenu la permission d'ouvrir des écoles, les Lazaristes ont créé l'école Saint-Louis à Téhéran en 1860, celle-ci était réservée aux filles; les sœurs Saint-Vincent de Paul ont fait de même en 1865 dans quelques villes de province. Au seuil du XX $\mathrm{XX}^{\mathrm{e}}$ siècle, les nouvelles écoles, comme Jeanne d'Arc et l'Alliance Française à Téhéran, ont agi remarquablement pour la propagation de l'enseignement de la langue et culture française dans la société iranienne.

La mainmise des Anglais et des Américains, à partir du début du $\mathrm{XX}^{\mathrm{e}}$ siècle, sur les affaires du pays a entraîné le recul progressif du français. À la suite de la visite officielle du Général de Gaulle en Iran, en 1963, les deux gouvernements se sont mis d'accord pour construire une autre école française à Téhéran appelée Zakariyyâ Razi du nom du célèbre

${ }^{1}$ La dynastie Qadjar (ou Kadjar) règne sur l'Iran de 1786 à 1925. 
médecin iranien du $\mathrm{X}^{\mathrm{e}}$ siècle. Mais les écoles citées plus haut ayant dû mettre fin à leur activité en Iran en 1979, le recul du français s'est accéléré et a cédé de plus en plus la place à l'anglais. Le français n'étant pas entré dans le pays par le biais de la colonisation, comme en Afrique, il est resté la langue préférée d'une certaine couche sociale privilégiée dans la société iranienne.

Par conséquent, l'enseignement du français, initié au XVII ${ }^{\mathrm{e}}$ siècle, atteignant son sommet au XIX ${ }^{\mathrm{e}}$ siècle avec l'ouverture du Dâr ol-Fonun, a fait marche arrière au début du $\mathrm{XX}^{\mathrm{e}}$ siècle, et plus encore après la Deuxième Guerre mondiale. Pourtant, la francophonie connait à la fin du $\mathrm{XX}^{\mathrm{e}}$ et au début du $\mathrm{XXI}^{\mathrm{e}}$ siècle (entre 1990 et 2010) un nouveau regain dans le pays. Actuellement, le français est enseigné dans vingt départements universitaires publics et privés dans les grandes villes au niveau de la licence, de la maitrise (Master II), et du doctorat. Au niveau de la licence, il y a deux filières : langue et littérature française et traductologie. Au niveau du Master II, il y a quatre filières: littérature, didactique, traductologie et plus récemment les études françaises. Au niveau du doctorat, il existe deux filières : la littérature française et comparée, et depuis l'année dernière (2010), la didactique du FLE. Le français s'enseigne au niveau secondaire dans la capitale et dans quelques grandes villes de province. En plus, une demande croissante pour le français se fait sentir depuis une dizaine d'année, dans les instituts privés, en particulier, pour ceux qui veulent se rendre dans les pays francophones, surtout au Canada, pour s'y installer ou pour poursuivre des études universitaires.

\section{La didactique du français en Iran}

La didactique du français constitue la partie intégrante de la francophonie, et c'est elle qui permet d'assurer sa survie et son développement. Dans cette partie, nous tenons à présenter la situation actuelle de l'enseignement du français en Iran, en vue de donner une perspective de son épanouissement. Il est à signaler que notre objectif dans cet article ne consiste pas à faire une étude purement didactique sur la méthodologie de l'enseignement du FLE en Iran. Nous nous contentons d'en faire un état des lieux sans aborder la relation pédagogique des trois éléments de Germain (10-14).

\section{A. Enseignant}

Dans les universités, les professeurs iraniens enseignent le français aux apprenants adultes. Acceptant l'autodidaxie chez les adultes, les enseignants doivent jouer le rôle de facilitateurs de l'apprentissage (Knowles 54). Le professeur de langue étrangère est tenu de mettre en œuvre toutes ses possibilités pour sécuriser le climat de la classe, une tâche qui est aussi valable pour un public enfant qu'adulte. Les professeurs de langue étrangère jouent un rôle notable pour décontracter les apprenants adultes. Lanchec constate aussi à ce propos que «l'efficacité d'une méthode moderne est en relation directe avec, d'une part, la formation du professeur et, d'autre part, le rayonnement de sa personnalité » (59). Ce que l'on oublie en Iran, c'est que, pour un professeur de FLE, les compétences linguistiques et phonétiques sont aussi prépondérantes que sa connaissance en psychologie et en psycholinguistique. 
Il ne faut pas oublier que les professeurs d'aujourd'hui étaient les étudiants d'antan, que les problèmes ne surgirent pas soudainement, et que la continuité en série et persistante de ces lacunes avait pour conséquence quelques défauts. Si on ne pratique pas la langue étrangère avec les autochtones, on risque de l'oublier, au moins au niveau oral. La présence des Français et francophones dans le corps professoral est, nous semble-t-il, indispensable, comme cela était autrefois le cas. Faute de quoi, la capacité des professeurs au niveau oral diminue, et ils préfèrent alors ne plus enseigner des cours utilisant l'audiovisuel qui exigent beaucoup d'efforts oraux. Ainsi, les professeurs se sont servis des mêmes textes littéraires pour leurs cours de littérature et traduction pendant des années. Pour exercer vraiment le métier de professeur d'une langue étrangère, le stage de recyclage et le séjour linguistique temporaire sont vitaux. Cette nécessité est primordiale, puisque les professeurs, pour construire leur savoir, sont tenus de faire leur remise à niveau, d'être au courant des nouveautés méthodologiques et des publications récentes des ouvrages spécialisés.

\section{B. Apprenant}

Nous agencerons nos réflexions autour de quelques paramètres concevables concernant l'apprenant iranien du FLE. Le public auquel nous nous adressons est celui qui vient à l'université, avec une moyenne d'âge de dix-neuf à trente ans. Il s'agit donc d'un public de jeunes adultes. Dans un cours de langue dispensé aux adultes, il faut d'abord chercher à appréhender cet «adulte» à travers sa personnalité et ses caractéristiques. La définition psychologique concerne directement notre domaine et peut apporter de multiples réponses à des questions didactiques. Selon M. Knowles, "nous devenons psychologiquement des adultes lorsque nous avons nous-mêmes pris conscience que nous sommes responsables de nos vies et que nous devons nous autogérer» (69). Nous considérons l'apprenant iranien du FLE comme celui qui possède ces particularités.

L'effet de l'âge renforce l'interférence de la langue maternelle et implique un certain effort supplémentaire pour acquérir la langue étrangère (le français) ; autrement dit, "les interférences dues à la langue 1 seraient plus fortes chez les adultes et (...), de manière générale, l'apprentissage d'une L2 serait d'autant plus difficile qu'on est plus âgé » (Gaonac'h 140).

La plupart du temps, l'apprenant iranien est « débutant complet », c'est-à-dire qu'il ne connaît pas du tout le français, lors de son entrée à l'université; ce fut exactement le cas des auteurs de ces lignes. Le besoin de visualisation (Lanchec 76) représente une autre particularité des apprenants iraniens du FLE. Il peut sembler qu'il est né des caractéristiques culturelles et, peut-être, de notre système éducatif antérieur en Iran. Il faut aussi rappeler que le système écrit de la langue persane en Iran est différent de celui de la langue française puisque on utilise l'alphabet arabo-persan. Par conséquent, pour un public adulte iranien débutant du FLE, qui, pourtant, a pris l'habitude de prendre des notes ou de visualiser la graphie des mots pour apprendre le sens et l'orthographe, on doit accorder une importance, même légère, à l'écriture, sans ignorer le risque de mettre en retard la prise de parole (Moirand 96). 


\section{Méthode}

Dans ce passage, nous tenons à exposer brièvement l'évolution des manuels du français en Iran depuis les trois dernières décennies sans vouloir comparer ou analyser des méthodes particulières, ce qui pourrait être une recherche à part entière. Il ne faut pas oublier que la «neutralisation du contexte» est nettement présente dans les méthodologies dites « universalistes » qui ne prennent pas en considération les variables culturelles (Béacco $8-15)$.

L'évolution des méthodes de français en Iran peut être suivie après la Révolution Iranienne. Après les vicissitudes et le bouleversement politiques qui ont secoué l'Iran, et contribué à l'avènement de la Révolution Islamique en 1979, toutes les infrastructures politiques, économiques, sociales et culturelles ont subi de profondes transformations. Dans cette atmosphère nouvelle, à l'image d'autres instances gouvernementales et nongouvernementales, les universités ont procédé à des changements dans leurs programmes. Il allait de soi que les disciplines comme celles des lettres et sciences humaines, et surtout, parmi elles, les langues étrangères qui concernaient de plus en plus l'Occident et la culture occidentale, devraient être modifiées. Avant la Révolution Islamique, toutes les méthodes de français enseignées en Iran étaient d'origine française. La charge culturelle de ces méthodes étant lourde, le nouvel univers révolutionnaire ne pouvait pas l'accepter. On considérait ces méthodes comme des instruments pour franciser et donc en général occidentaliser les apprenants.

Les méthodes les plus utilisées dans les universités iraniennes étaient La France en Direct, Voix et Images de France, De Vive Voix et le Cours de langue et civilisation française de Manger. Cette dernière était enseignée depuis une cinquantaine d'années en Iran. À l'occasion de la Révolution Culturelle qui entre autres entraina la fermeture des universités de 1980 à 1982. Les professeurs de français iraniens furent chargés de l'élaboration de nouvelles méthodes contenant moins d'aspects culturels et étant plus conformes aux réalités de la nouvelle société iranienne. Ils ont alors adopté une stratégie d'adaptation culturelle qui n'était pas de supprimer entièrement la culture et la littérature française dans les nouvelles méthodes. Ils ont réussi, après avoir surmonté de nombreux écueils, à publier depuis 1984, une trentaine d'ouvrages d'enseignement du français dédiés à l'apprentissage de la langue et aussi de la littérature française et francophone comme par exemple Initiation à la négritude : anthologie. Ces ouvrages sont soit bilingues soit intégralement en français.

Malgré tout, depuis une quinzaine d'années, on assiste à une nouvelle tendance consistant à utiliser les nouvelles méthodes de publication française. Aujourd'hui, les méthodes plus récentes comme Alter Ego, Campus, Mosaïque sont utilisées dans les universités iraniennes.

\section{Une francophonie latente}


Sans tenir compte du nombre restreint des francophones iraniens (la majorité est constituée de diplômés de langue française des universités iraniennes), le français ne se pratique pas dans la société. La francophonie latente comprend alors au moins deux postulats linguistique et culturel.

\section{A. Francophonie linguistique latente : le cas de l'emprunt et du calque}

La francophonie linguistique latente en Iran peut s'expliquer par le fait qu'il existe un certain nombre de mots et d'expressions français que les Iraniens utilisent couramment dans leurs échanges quotidiens, soit avec une prononciation proche du français, soit avec une prononciation persanisée, sans qu'ils sachent leur origine.

L'histoire de l'insertion des mots français remonte au milieu du XIX ${ }^{\mathrm{e}}$ siècle lorsque le mouvement de la traduction a été lancé en Iran pour accéder aux ouvrages des écrivains européens. La majorité imposante des ouvrages dans différents domaines étaient en français. La raison en est bien claire : à cette époque, comme nous l'avons vu, la cour royale et de nombreux intellectuels parlaient français. Ces derniers ont commencé à traduire des livres français non seulement dans leur spécialité, mais aussi dans différents domaines. Ce mouvement s'est accéléré avec la Révolution Constitutionnelle de 1906. Grâce à la traduction des grands écrivains tels Descartes, Voltaire, Montesquieu, Rousseau, Racine, Hugo, les lecteurs iraniens se sont initiés à la littérature, à la philosophie et à la culture française. C'était le début de la réception des ouvrages français. Ainsi, la langue, la littérature et la culture française ont imprégné la langue et littérature persane par la traduction.

Comme la porte d'entrée principale de la francophonie en Iran était la traduction, on peut chercher à étudier sa trace dans la langue persane sous la forme de l'emprunt et du calque. Ainsi le nombre des livres traduits du français en persan est plus nombreux que celui des autres langues ; c'est pourquoi aujourd'hui, les emprunts français en persan sont beaucoup plus nombreux que ceux des autres langues. Les emprunts lexicaux sont des solutions pour une lacune lexicale scientifique, technologique ou culturelle dans la langue emprunteuse (Ladmiral 19). Les emprunts d'origine française concernent toutes les catégories linguistiques; la langue courante, scientifique, politique, journalistique, littéraire, etc. Comme la liste est assez longue, nous en donnons à titre d'exemple quelques-uns qui concernent des mots de la langue de tous les jours comme "carte », "cravate ", "merci », "banque », " téléphone », " asphalte », «tableau», " lustre », " conférence », " classeur », "carton», "commission", «standard» et des termes des domaines de spécialité comme « budget», «bilan», «vaccin», « microbe », «stratégie », " phonétiques », «bourse », « régime», « diplomatie », " genre» (littéraire), «scène », « mise en scène », " personnage », « clinique », " seringue », « pompe», «bandage ». Ces emprunts subissent parfois des changements phonétiques. En ce qui concerne le calque, on peut donner des exemples comme " chemin de fer", " compter sur qqn ", "monter sur la scène ", "points de vue ", "premier ministre », "ministère des affaires étrangères ", "lettre ouverte », « liberté politique », " grève générale », « coup d'état », « force majeure ». Comme la plupart des mots empruntés au français sont naturalisés et familiers, même certaines propositions 
de remplacement venant de l'Académie de la langue persane (fondée en 1935) n'ont pas trouvé d'accueil favorable dans le public iranien.

\section{B. Francophonie culturelle latente}

La situation sociolinguistique actuelle de l'Iran est marquée par l'unanimité de la langue officielle, le persan. Néanmoins, plusieurs langues vernaculaires et en situation diglossique (Neveu 57 \& 303) se trouvent dans tout le pays. En effet, aucune langue d'une portée internationale comme l'anglais ou le français ne joue un rôle communicatif dans ce contexte. L'histoire de l'Iran a montré qu'aucune langue autre que la langue persane considérée comme l'héritage culturel et identitaire le plus cher des Iraniens - n'est devenue une langue véhiculaire .

Cette langue, longtemps considérée en Iran (1860-1930), comme véhicule de l'enseignement des matières philosophiques, techniques et scientifiques, n'est pas actuellement la seule langue véhiculaire de ces domaines. Il faut rappeler que les paradigmes intellectuels occidentaux, présents au sein des évolutions des sciences sociales et humaines en Iran, de nos jours, sont encore d'un statut dichotomique : anglophone/francophone.

De nos jours, à l'heure des réalités linguistiques changeantes, la langue française se trouve de plus en plus perçue comme une langue d'ouverture sur le monde (ou à tout le moins une partie importante du monde), une langue de la modernité et de la culture. C'est là également dans la société des gens de culture, que la langue française continue, contre vents et marées, à jouir d'un statut privilégié. En effet, les changements politiques et les politiques d'enseignement n'ont pas été influents sur ce statut particulier du français. Un aperçu général sur ce sujet fortifie le postulat d'une francophonie culturelle latente dont les engrenages remontent à son importance historique en Iran. Au milieu du XIX ${ }^{\mathrm{e}}$ siècle alors que les deux puissances politiques de l'époque, la Russie et l'Angleterre, étaient très impliquées dans les affaires politiques de l'Iran, c'étaient les écoles françaises qui enseignaient l'anglais et le russe. Certains justifient cet intérêt des lettrés iraniens pour le français par le fait qu'ils considéraient la France comme un allié contre les deux pouvoirs impérialistes voisins. Nous croyons que cela ne pourrait pas être acceptable, car cette tendance politique n'entraînait normalement pas une telle fascination pour la langue française. Par l'acquisition du français, ils souhaitaient ouvrir de nouvelles fenêtres vers le développement culturel. En effet, ils voyaient le français comme le sésame d'Ali Baba pour accéder à l'héritage des idées des Lumières, de la Révolution française et de la France moderne.

Pendant toute la période du $\mathrm{XIX}^{\mathrm{e}}$ et $\mathrm{XX}^{\mathrm{e}}$ siècle, la pensée française occupe entièrement le cœur des élites iraniennes. Bien que les œuvres les plus traduites et lues (par exemple, dans le domaine des lettres) ne soient pas forcément les plus connues en France ou dans le monde (par exemple, Roman Gary), le choix de ces œuvres en Iran est très influencé par la situation sociopolitique du pays. Les intellectuels iraniens cherchent dans chaque écrivain et penseur français, un combattant de la liberté et un auteur engagé. Ils 
suivent de plus près les mutations sociopolitiques et les évolutions culturelles de l'aire française. L'affaire Dreyfus, par exemple, marqua beaucoup l'intelligentsia iranienne et finalement l'acquittement de ce dernier, en juillet 1906 - année de la Révolution Constitutionnelle en Iran - fut très prometteuse et encourageante pour les Constitutionnalistes iraniens.

En effet, ce mouvement engagé pour la traduction de l'héritage des Lumières cherche une nouvelle conscience politique qui vise avant tout des solutions pour les problèmes de la société. Même dans un établissement technocratique comme Dâr-olFonoun, outre les ouvrages techniques et didactiques, la première génération des étudiants traducteurs iraniens, s’intéressa à la traduction littéraire et surtout à celle des romans historiques. Les lecteurs iraniens se familiarisaient ainsi avec la culture, l'histoire et la pensée française. Les hommes de lettres, à leur tour, découvrait une nouvelle vision de l'Histoire et un nouveau genre littéraire (le roman), caractérisé surtout par la simplicité du langage par rapport à la langue complexe, alambiquée et artificielle en vogue parmi les gens de lettres de la période précédant la Révolution Constitutionnelle. Cette familiarisation avec la littérature française dans l'évolution de la prose et de la poésie de l'Iran contemporaine constitue une partie cruciale de l'Histoire littéraire du pays. Parmi les tendances thématiques et structurelles, on peut énumérer brièvement des mouvements et des écoles déduits de cette familiarisation : poésie et journalisme engagés, récits historiques et autocritiques, situation féminine (1890-1930), nouvelle prose littéraire, thématique sociale et romantique dans les magazines et les journaux parfois bilingues (persan-français) publiés à l'intérieur et à l'extérieur du pays, exil d'un cercle des écrivains et poètes à Paris (19301950), regard cosmopolite, modernité, nouvelle vague dans la poésie et la nouvelle après 1950 (Azhand 25-55).

\section{Francophonie latente de l'Iran d'aujourd'hui}

Nous croyons qu'en Iran aujourd'hui, si le français a des usages de plus en plus limités, il n'est pas en déclin. Le bilinguisme persan-français est la possibilité pour un locuteur universitaire de recourir, en fonction de la situation de communication académique et interculturelle, à deux langues différentes pour enrichir ses acquisitions et ses productions. Par ailleurs comme ce bilinguisme persan-français a trouvé des tendances renouvelées pour s'approcher du «multilinguisme ou plurilinguisme » (Charnet 195), d'autres bilingues universitaires comme en persan-anglais développent un nouveau penchant pour l'apprentissage d'autres langues et entre autres du français .

La tradition de polyglottisme, familière sous d'autres climats, est devenue pour la plupart des partisans des sciences humaines et sociales une question importante en Iran. Un sujet polyglotte émerge au sein des établissements attachés aux sciences sociales et humaines : un universitaire polyglotte est un vrai érudit dans son domaine de recherche. Alors, la nécessité d'étudier dans le texte original et ses postulats langagiers ne cesse de hanter l'esprit de tous ceux qui se montrent soucieux d'acquérir et de représenter authentiquement les savoirs en sciences humaines. 
Le paysage linguistique du français en Iran se distingue par le fait que de nouveaux préceptes divers s'enchevêtrent pour donner lieu à une situation linguistique plus complexe. Les nouvelles mutations exigées pour les sciences humaines (islamisation et «naturalisation »/nativisme) commandées par le discours officiel du pays, participeraient à faire de cette situation un terrain linguistique qui échappe à toute analyse qui se veut définitive. Dans les domaines comme la sociologie, la philosophie ou le droit, il y a une sorte de consensus sous-jacent à ce que non seulement la francophonie culturelle latente ne se décrédite pas, mais aussi à ce qu'elle s’impose davantage.

Un autre facteur social qui doit être ajouté à ce contexte est le phénomène de l'immigration actuelle vers les pays francophones. Aujourd'hui, la part du lion pour le nombre d'immigrés iraniens revient au Canada francophone et à la France. Cette communauté iranienne vivant dans ces deux pays est remarquablement une communauté d'universitaires, artistes et intellectuels. La francophonie devient, pour cette communauté qui fréquente couramment le pays d'origine et les pays d'accueil, un élément influent de l'identité sociale.

L’ensemble de la présence scolaire, pédagogique, éditoriale (Presses Universitaires, Revue de Téhéran, Institut Français de Recherche en Iran-IFRI) et médiatique francophone en Iran (Chaîne Internationale Sahar et Radio francophone iraniennes) n'est pas d'une grande hétérogénéité. C'est une francophonie universitaire, artistique et intellectuelle qui suit des volontés typiquement culturelles et intellectuelles de curiosité et de séduction interculturelles.

Le choix de nouvelles générations francophones iraniennes pourrait être toujours dans le sens de «l'unité dans la diversité ». En effet, « la Francophonie vivra si sont réussies ses noces avec la langue française », et «un français qui n'est pas 'une tour' ou une 'cathédrale', mais une passerelle destinée à faire mieux communiquer entre elles les civilisations qui se partagent le monde » (Jouve 400). Ce n'est donc pas simplement un espoir de perpétuité pour la langue de l'Hexagone ou un regroupement sociopolitique. C'est parce que l'élite iranienne, dont la grande partie des soucis est toujours la culture dans tous ses états, croit que la réalisation d'un nouvel équilibre universel passe nécessairement par l'affermissement et la collaboration de grands espaces paradigmatiques, culturels et linguistiques .

L'importance de la diversité linguistique et culturelle face aux défis de la mondialisation est parmi ses vraies questions ouvertes. L'élite iranienne croit que la France contemporaine en particulier et le monde francophone en général ne semblent pas souffrir d'un « déficit d'idées». À son sens, le problème ce n'est pas l'anglais, c'est le monolinguisme, c'est le prêt-à-porter culturel et l'uniformité hégémonique. Même très minoritaires, les gens de ce cercle implicite, croient très sincèrement qu'ils ne sont pas simplement dans une forteresse assiégée, à défendre un tout petit pré-carré. Ils se considèrent comme les porte-paroles de toutes les cultures du monde qui peuvent se sentir menacées par le monolinguisme croissant. Il leur semblerait donc pertinent de former ensemble un vrai réseau de coopération et de solidarité linguistique, culturelle et 
scientifique efficace et d'attester constamment sa dimension polyphonique, humanisante, démocratique et dialogique dans un monde globalisé où les chocs culturels se multiplient.

Pour l'élite iranienne, cet alliage, qui fait de la langue et de la pensée francophone un équivalent de sens, d'esprit de valorisation, de dignité, de créativité et de partage, est un appel au respect d'autrui et à une manière d'être au monde. Elle croit que ce message culturel garantit la possibilité du dialogue, la liberté d'expression, défend le droit de l'accès aux savoirs et aux expertises, encourage le débat d'idées et soutient le sens et la pratique de la démocratie, la diversité des langues et la possibilité de leur traductibilité en termes de langages, mais aussi en termes d'interaction et d'acculturation mutuelle entre les peuples et leur chance pour refaire le monde.

Grace à cette élite francophone qui a joué un rôle important dans la vie socioculturelle du pays et qui a conservé l'usage de cette langue, la francophonie culturelle latente est aujourd'hui encore considérée comme un instrument privilégié de formation intellectuelle, d'échanges, d'accès à l'information et à la connaissance, en particulier dans certains domaines comme les études culturelles, le droit, la sociologie, l'art, etc. Le souvenir, toujours vivace, de grands intellectuels, traducteurs, écrivains et poètes francophones iraniens, entretien l'image positive du français comme langue de la pensée et de la culture. Cette image constitue une base solide pour relancer la pratique de cette langue, et pour lui assurer une place à côté de l'anglais, indubitablement avantagé par son statut de « langue des affaires ».

\section{Réception de la francophonie littéraire en Iran}

Comme nous l'avons présenté, l'état actuel de la francophonie en Iran est très lié à un paradigme culturel. Pour éviter d'élargir notre corpus aux domaines artistique et cinématographique, nous aborderons seulement le domaine de la littérature et ses études. La littérature contemporaine et extrême-contemporaine française continue à être traduite et cela parfois par des disciples des traducteurs dont la renommée remonte aux tentatives de présenter les grands écrivains français $\mathrm{du} \mathrm{XX}^{\mathrm{e}}$ siècle. Nous pouvons énumérer, entre autres, Gide (traduit par Hassan Honarmandi), Camus et Sartre (Jalal Al Din Alam), Malraux (Abolhassan Najafi et Réza Seyedhosseini), Proust (Mehdi Sahabi), etc. Il faut noter que les études traductologiques qui se réalisent de nos jours sont majoritairement imprégnées par les grands noms de cette lignée des traducteurs francophones comme Mohammad Ghazi, Behazin et le grand intellectuel iranien Jalal Al-e Ahmad.

Quant aux autres littératures francophones comme celles de l'Afrique, considérées autrefois comme "périphériques ou mineures», elles ont commencé à obtenir une attention particulière, et leur réception est souvent prise comme sérieuse dans le champ littéraire et dans les domaines de recherche. Nous croyons que dans une société comme celle de l'Iran d'aujourd'hui, se trouve une tendance qui veut espérer créer, par l'émergence de cette nouvelle conception de la francophonie latente, un antipode à «la gravité du processus de différenciation voire de marginalisation qu'ont subit ces littératures dans le domaine du savoir et de la connaissance » (Fayol 1979 : 217). 
Au sein des départements de français dans les universités iraniennes, il y a une unité de valeur intitulée «initiation à la lecture des textes des écrivains francophones ». De même, dans les autres établissements universitaires et dans les différents départements comme les départements de littérature persane, de traductologie, de sociologie et d'anthropologie, il y a une percée analytique et conceptuelle considérable qui se manifeste de plus en plus par des nouvelles études interculturelles. En ce qui concerne les études comparées ou postcoloniales, l'application de diverses approches théoriques au corpus littéraire francophone se manifeste par un grand penchant des chercheurs et des étudiants iraniens envers les auteurs francophones d'origine iranienne comme Yasmina Reza et Marjane Satrapi, ou l'afghan persanophone ${ }^{2}$ Atigh Rahimi ou d'autres nationalités comme Amin Malouf, Assia Djebar, Ahmadou Kourouma, Tahar Ben Jelloun, Ismail Kadaré, Aimé Césaire, Frantz Fanon, Kateb Yacine et beaucoup d'autres.

Malgré leurs différences, ces travaux explorent des voies convergentes en relation avec cette conception de la francophonie latente. En effet, il est permis de croire à une conscience commune qui rend particulièrement précieuse la dimension démocratique et dialogique de la recherche littéraire. (Payandeh 2007 : 11) D'ailleurs, la littérature iranienne est très souvent soumise à des méthodes de lecture inspirées de la "nouvelle critique française et francophone » très à la mode. Un regard rapide sur les bibliographies des publications, soit universitaires, soit en dehors des universités, où il y a des travaux monumentaux comme La structure et le commentaire herméneutique du texte (Sakhtar va tavil-e matn) de Babak Ahmadi, prouverait cette influence.

Nous croyons que ce goût pour la francophonie latente dans l'enseignement, la lecture et l'interprétation littéraire en Iran se justifie par un vrai potentiel de convergences des discours socioculturels des textes francophones et les infrastructures socioculturelles de la société iranienne. En l'occurrence, ils ont beaucoup de points communs dans les motifs et les thèmes concernés, la structure générale des écrits, les élans de la pensée, la genèse du départ, et le comment de l'exposition. Il apparaît que les textes francophones présentent, un des plus riches, des plus beaux et des plus complexes répertoire thématique et stylistique, le fond des faits sociohistoriques, des caractéristiques et des contours culturels convergents comme le montre la réception des ouvrages tels Allah n'est pas obligé ou En attendant le vote des bêtes sauvage de Kourouma ou bien L'enfant de sable de Ben Jelloun en Iran.

Conçus pour être, la plupart du temps, des représentations véhémentes des crises et des malaises, de tels textes des « romanciers du réel » (Dubois 2000) constituent, y compris dans leur dimension esthétique, des témoignages authentiques sur les infrastructures sociales qui sont bien compréhensibles voire repérables et familières chez les interlocuteurs iraniens. Les relations que ces textes et leurs principales composantes contextuelles entretiennent avec la généalogie sociohistorique de la société iranienne, accentuent le fait que le sens référentiel des réalités de ces œuvres est commun. Autrement dit, cela

${ }^{2}$ Le persan est une langue parlée en Iran, en Afghanistan, et au Tadjikistan en tant que langue officielle, ainsi qu'au Bahreïn (minorité iranophone) et en Ouzbékistan (minorité tadjik). 
représente un sens qui sert d'appel à une fonction de «miroir magique». (Bellemin-Noël $1996: 76)$

En inaugurant le style du réalisme sociopolitique, un fabuleux hymne à la tradition, un coup de force stylistique de l'écrit et un réseau de références factuelles et fictionnelles d'images et de saveurs, ces textes ne cessent d'attirer l'attention des lecteurs iraniens qui leur permet de toucher du doigt des parties cruciales d'une Histoire et d'une Tradition à peu près communes. Leur écriture contestataire et dénonciatrice qui a toujours lancé des cris d'alarme et qui a manifesté une prise de distance avec le conformisme littéraire, découle effectivement d'une préoccupation profonde d'élaborer une signification révélatrice à la fois critique et pragmatique. Résidant alors dans l'art de saisir les aspects de la tradition, de la culture et de l'Histoire refoulés parfois dans la marge, le silence ou l'indifférence, leur originalité correspond à une perception épistémologique dans laquelle passé et présent s'entremêlent.

Il va sans dire que toutes ces contributions élargissent un champ favorable pour l'avancement de la francophonie latente en Iran, tant dans son contexte didactique, heuristique qu'herméneutique. En d'autres termes, nous croyons que cette dimension de la francophonie latente qui facilitera la portée polyphonique et dialogique des infrastructures discursives communes, serait simultanément amplifiée, et que son vecteur de cohérence des optiques culturelles serait pleinement assumé pour jouer un rôle non négligeable dans l'avenir.

\section{Conclusion}

La présence du français en Iran remonte au XVII ${ }^{\mathrm{e}}$ siècle. La survie du français est due aux efforts des premiers missionnaires français et des couches lettrées de la société de l'époque. Sa persistance est due à son enseignement dans les établissements universitaires et secondaires. L'efficacité de cet enseignement dépend des composantes de l'acte didactique, à savoir l'enseignant, l'apprenant et la méthode. L'enseignant, comme partout dans le monde, a besoin d'avoir des échanges avec les natifs français dans le corps professoral, ce qui fait défaut aujourd'hui. La majorité des apprenants iraniens sont adultes et débutants complets qui sont habitués à visualiser et à écrire pour apprendre le français. En ce qui concerne la méthode, il faut dire que le mouvement de neutralisation culturelle des méthodes de publication française n'affecte guère le processus de l'apprentissage du FLE en Iran. De nos jours, on assiste à une augmentation importante des demandes pour apprendre le français pour des raisons différentes. La didactique du français a joué et continue à jouer le rôle de soutien et de garant de la francophonie latente en Iran.

Si le statut particulier du français en Iran n'est pas nettement défini, la question de la francophonie est une question qui touche encore l'esprit des universitaires et des élites iraniens. Bien que cette élite ne tienne pas majoritairement à choisir le français pour écrire et parler, cette langue n'est pas aussi étrangère que d'autres car elle jouit d'un statut particulier. Ce statut, ambivalent, tient compte de facteurs intrinsèques aux valeurs 
culturelles véhiculées par la langue française auxquelles les concepteurs/récepteurs de la société contemporaine de l'Iran adhèrent d'une manière ou d'une autre.

S'apercevant que le statut de la langue française en Iran ne serait pas restreint dans les acceptions coutumières de la francophonie, nous avons cherché à caractériser les traits spécifiques d'une francophonie latente qui émergeait au moins dans deux sphères linguistique et culturelle. Pour le côté de la francophonie linguistique latente, nous voulions mettre en évidence la présence d'un grand nombre d'emprunts et de calques parfois entièrement naturalisés au sein du persan. En ce qui concerne la francophonie culturelle latente, nous tenions à montrer qu'elle continue à préserver des traces très saillantes dans les domaines intellectuels et interculturels de l'Iran.

Nous croyons qu'une telle vocation interculturelle de la francophonie peut contribuer à une nouvelle vision de la culture internationale qui considère les traditions comme traductibles, interprétables et partageables. Cette conception de la francophonie culturelle et linguistique latente résistera contre l'universalisme qui banalise les traditions culturelles, contre le communautarisme qui juxtapose les entités sociales et culturelles, et qui ne respecte pas la diversité des expressions culturelles. Il apparaît que la minorité francophone ou même la francophilie de l'Iran, dont une grande partie de son élite d'hier et d'aujourd'hui s'est enorgueilli, ne pourrait pas éviter le fait que la francophonie linguistique et culturelle latente est un outil essentiel et nécessaire de formation, d'information et de développement, et qu'elle dispose d'atouts incontestables pour promouvoir l'avènement d'un monde meilleur. Cette francophonie qui se situe dans une perspective réaliste et équilibrée est celle du développement de la connaissance du français considéré comme un atout supplémentaire mais privilégié pour tous ceux qui le perçoivent comme une langue offrant l'accès à des formations/informations interculturelles et des savoirs de qualité.

Pour toutes les raisons évoquées et pour d'autres encore, la situation de la francophonie latente demeure en Iran à la fois privilégiée et aléatoire. Jouissant d'un statut particulier sur les plans des différents secteurs de la culture, son statut favorisé continue à laisser des traces très délicates et omniprésentes dans l'ambiance culturelle de la société iranienne, surtout chez les hommes et les femmes de culture, d'art et de médias, les experts universitaires, les écrivains et les poètes. Bien que ce statut soit incertain pour les uns, il sera une chance pour les autres, leur permettant d'aller vers de nouveaux horizons d'ouverture et d'échange s'inscrivant dans une immense " participation à une signification commune ». (Gadamer 313) 


\section{Bibliographie}

Ahmadi, Babak. La structure et le commentaire herméneutique du texte (Sakhtar va tavil-e matn). Téhéran : Édition Markaz, 2002.

Azhand, Yaghoub. La littérature moderne d'Iran (Adabiat-e novin-e Iran). Téhéran : Editions Amir Kabir, 1986.

Béacco, Jean-Claude. «L'intervention didactique et les variables culturelles ». $\mathrm{N}^{\circ}$ spécial du Français dans le Monde. 1996.

Bellemin-Noël, Jean. La psychanalyse du texte littéraire. Paris : Nathan, 1996.

Charnet, Chantal. in Détrie, Catherine, Siblot, Paul, Verine, Bertrand. Termes et concepts pour l'analyse du discours. Une approche praxématique. Paris : Honoré Champion, 2001.

Dieulafoy, Jane. Une amazone en Orient: Du Caucase à Persépolis 1881-1882. Paris : Éditions Phébus, 2010 .

Dubois, Jacques. Les Romanciers du réel. De Balzac à Simenon. Paris : Le Seuil, 2000.

Fayol, Roger. «Quelle sociocritique pour quelles littératures ». Sociocritique. Paris : Ferdinand Nathan, 1979.

Gadamer, Hans-Georg. Vérité et Méthode. Paris : Le Seuil, 1996.

Gaonac'h, Daniel. Théories d'apprentissage et acquisition d'une langue étrangère. Paris : CREDIF, Hatier, Coll. L.A.L, 2004 .

Germain, Claude. Evolution de l'enseignement des langues: 5000 ans d'histoire. Paris : CLE international, 2004.

Girard, Denis. Linguistique appliquée et didactique des langues. Paris : Éd. Armand ColinLongman, $4^{\mathrm{e}}$ Édition, 1972 .

Grand Dictionnaire Terminologique (GDT). http://www.granddictionnaire.com /btml/fra/r_motclef/ index800_1.asp.17/8/2012.

Houssaye, Jean. La pédagogie: une encyclopédie pour aujourd'bui. Paris : ESF éditeur, coll. Pédagogie, 2009.

Jouve, Edmond. Relations internationales. Paris : PUF, 1992.

Keyhani, Sadegh. "Petite histoire de l'enseignement de la langue française en Iran » Revue électronique de l'Association Culturelle Franco-Iranienne Sabalan. Mars 2005.

Knowles, Malcom Shepherd. L'Apprenant adulte, vers un nouvel art de la formation. Paris : Les Editions d'Organisation, traduit par F. PABAN, 1995.

Ladmiral, Jean-René. Traduire: théorèmes pour la traduction. Paris : Gallimard, 2010.

Lanchec, Jean-Yves. Psycholinguistique et pédagogie des langues. Paris : P.U.F, coll. S.U.P., 1976.

Moirand, Sophie. Situation d'écrit: compréhension et production en français langue étrangère, Paris : CLE International, 1990 .

Neveu, Franck. Dictionnaire des sciences du langage. Paris : Armand Colin, 2004.

Payandeh, Hossein. La Critique littéraire et la démocratie (Naghd-e adabi va démocrasi). Téhéran : Éditions Niloufar, 2007.

Tavernier, Jean-Baptiste. Les six voyages en Turquie et en Perse. Paris : Librairie François Maspero, 1981. 